\title{
HISTÓRICO DE QUEDAS, CAPACIDADE FUNCIONAL E AUTOEFICÁCIA EM IDOSAS COMUNITÁRIAS: UMA ANÁLISE COMPARATIVA
}

\author{
Bruno Sousa LOPES \\ Gustavo Azevedo CARVALHO² \\ 1 Fisioterapeuta, mestrando em Gerontologia pela Universidade Católica de Brasília (UCB), e-mail: \\ brunoslopesfisio@gmail.com. Autor para correspondência. Endereço: SHIN QI 04 Conjunto 07 Casa 05, Lago Norte. \\ Brasília - DF - Brasil \\ ${ }^{2}$ Fisioterapeuta, doutor em Ciências da Saúde pela Universidade de Brasília (UNB), professor titular do curso de \\ fisioterapia da Universidade Católica de Brasília (UCB), Brasília, DF - Brasil, e-mail: carvalhobsb@hotmail.com
}

Recebido em: 20/05/2014 - Aprovado em: 30/06/2014 - Disponibilizado em: 30/07/2014

\begin{abstract}
Resumo: Existem alterações fisiológicas que o ser humano sofre com o passar dos anos. Tais mudanças afetam a funcionalidade e a autoeficácia do idoso, tornando-o mais suscetível a quedas e consequentemente a fraturas. Comparar a capacidade funcional, medo de cair, autoeficácia e histórico de quedas em idosas caidoras e não caidoras e analisar descritivamente as estratificações de riscos de quedas. 41 idosas participaram do estudo, sendo avaliada a funcionalidade, através dos testes de Alcance Funcional (TAF); Alcance Lateral (TAL); Escala de Equilíbrio de Berg (EEB), medo de cair pela Falls Efficacy Scale - Internetional adaptado culturalmente para a população brasileira (FES-I-Brasil) e o histórico de quedas. Variáveis foram comparadas entre os grupos caidoras e não caidoras. Análise descritiva dos escores de estratificação e teste de correlação das variáveis no grupo total. Ao dividir o grupo em caidoras (18) e não caidoras (23) foi encontrado comparação entre o TAF. No grupo total observou-se correlação entre idade e EEB; número de quedas no último ano e TAF; TAF e EEB; TAL e EEB. TAF foi o melhor teste para diferenciar os grupos; TAF, TAL e EEB demonstram resultados semelhantes, porém um não deve substituir o outro. A EEB pode apresentar diferentes resultados, dependendo do nível de senescência.
\end{abstract}

Palavras-chave: Idoso. Acidentes por quedas. Autoeficácia. Equilíbrio postural. Envelhecimento.

\section{FALLS HISTORY, FUNCTIONAL CAPACITY AND SELF EFFICACY IN OLDER COMMUNITY: A COMPARATIVE ANALYSIS}

\begin{abstract}
There are physiological changes that human being suffers over the years. These changes affects the functionality and self efficacy in the elderly, making him more susceptible to falls and therefore fractures. To compare the functional capacity, fear of falling, self efficacy and history of falls in elderly fallers and non-fallers and analyze descriptively the stratifications risk of fall. 41 elderly participated in the study, assessing functionality using the tests Functional Reach (FRT); Lateral Reach (LRT); Berg Balance Scale (BBS), fear of falling by the Falls Efficacy Scale International Culturally adapted for the Brazilian population (FES-I-Brazil) and history of falls. Variables were compared between groups of fallers and non-fallers. Descriptive analysis of scores stratification and test of correlation of the variables in the whole group. When dividing the group between fallers (18) and non-fallers (23) was found comparison between the FRT. The total group correlation was found between age and BBS; number of falls in the last year and and FRT; FRT and BBS; LRT and BBS. FRT was the best test differentiate the groups; FRT, LRT and BBS demonstrated similar results, but one should not replace the other. BBS may show different results, depending on the level of senescense.

Keywords: Elderly. Accidental falls. Self efficacy. Postural balance. Aging.
\end{abstract}




\section{INTRODUÇÃO}

As alterações irreversíveis que acontecem, ao passar dos anos, de uma forma contínua e lenta na morfologia de diferentes células, tecidos e órgãos exercem um impacto diretamente na capacidade funcional do indivíduo, podendo se agravar com o sedentarismo. A força, o equilíbrio, a flexibilidade, a agilidade e a coordenação motora constituem variáveis afetadas pelo processo de envelhecimento fisiológico do ser humano (GONÇALVES et al., 1990; NÓBREGA et al., 1999; TEIXEIRA; GUARIENTE, 2010).

É descrito que indivíduos na faixa etária a partir dos 50 anos enfrentam problemas como a redução da força, que pode chegar a $10 \%$ de perda por década (NÓBREGA et al., 1999). A redução da capacidade muscular na terceira idade está relacionada com a sarcopenia, um processo fisiológico do ser humano que leva a redução involuntária da massa e força muscular, ocorre principalmente por redução das fibras musculares do tipo II, de contração rápida. (DOHERTY, 2003; OCHALA et al., 2006; WALRAND et al., 2011).

Concomitantemente, essa redução da massa e força muscular afetam a funcionalidade do idoso, causando limitações como mobilidade reduzida, diminuição da densidade óssea e fraturas pelo aumento do risco de quedas. (LINDLE et al., 1997; NÓBREGA et al., 1999; WALRAND et al., 2011). Gonçalves (2010) afirma em seu estudo que é possível que os idosos adquiram independência para realizar atividades do cotidiano, e que para isso é necessário que tenham boa coordenação, agilidade e equilíbrio dinâmico, ou seja, uma boa aptidão física, possibilitando um desempenho funcional adequado, contribuindo assim para melhorar a qualidade de vida do indivíduo (HYATT et al., 1990; ROSA et al., 2003).

As condições psicológicas também podem influenciar diretamente as aptidões físicas e consequentemente a funcionalidade, tornando-se um fator de risco para quedas. Dentre estes fatores psicológicos o medo de cair pode ser um limitante na mobilidade de idosos, levando-os a adotar uma vida com pouco contato social. Além de provocar restrições de movimento, alterações de equilíbrio e controle postural o medo pode levar os indivíduos a uma redução de condicionamento físico, sendo caracterizado como risco de quedas (WALKER; HOWLAND, 1990; FLETCHER; HIRDES, 2004).

Sabe-se que os maiores gastos com idosos no sistema de saúde do Brasil estão relacionados com fraturas decorrentes de quedas, já que é necessário investir em procedimentos cirúrgicos, internações, materiais protéticos e medicamentos 
antibióticos. A queda em idosos se torna um causador de sérios prejuízos à qualidade de vida dessa população, podendo influenciar na mobilidade, gerar dependência dos familiares, além dos altos índices de mortalidade póscirúrgico (ARAÚJO; OLIVEIRA; BRACCO, 2005; CHRISTOFOLETTI et al., 2006; GUIMARÃES et al., 2004).

Notando os diversos problemas econômicos e sociais em virtude de quedas, é necessário aprofundar cada vez mais nos estudos sobre esta população em risco para traçar planos de prevenção. Com isso, percebe-se a grande necessidade em constatar os fatores causais dos eventos de quedas e sua correlação com o déficit funcional, além da correlação deste déficit com o medo de cair, possibilitando então investir em medidas que resultem na diminuição de quedas e consequentemente na redução de gastos.

O objetivo deste trabalho é comparar a capacidade funcional, o medo de cair, a autoeficácia e os episódios de quedas das idosas caidoras e não caidoras avaliadas. Além disso, realizar análise descritiva dos resultados de estratificação dos riscos de quedas. Posteriormente, correlacionar estas variáveis no grupo total de idosas e abordar a importância da capacidade funcional e autoeficácia.

\section{MATERIAIS E MÉTODOS}

Foi realizado um estudo do tipo transversal, pois segundo Pereira (2008) este tipo de pesquisa retrata a relação entre variáveis, que no presente estudo abordou a autoeficácia, histórico de quedas e medo de cair, que foram coletadas com uma população especifica e em um exato momento.

Foram convidadas a participar do estudo idosas inscritas no programa Centro de Convivência do Idoso que atende 369 idosos nas diversas atividades sociais, físicas $\mathrm{e}$ escolares, tais como coral, artesanato, aprendizado de língua estrangeira e outras.

Para selecionar a amostra, a secretaria do CCI foi consultada a respeito dos locais onde o grupo de idosas desejado realizavam suas atividades. Ao localizar os indivíduos, os pesquisadores explicaram os objetivos, a metodologia do estudo, e realizaram um convite para participarem do mesmo. A amostra foi selecionada por conveniência, totalizando 41 idosas selecionadas. As idosas foram avaliadas para saber se não estavam dentre algum critério de exclusão, como histórico de lesões ou doenças ortopédicas, neurológicas e reumatológicas que influenciavam no procedimento de coleta dos dados; histórico de fratura no último ano anterior a coleta; queixa de quadro álgico que influenciava ou impossibilitava a realização dos testes propostos; anomalias congênitas ou adquiridas de membros superiores e membros inferiores; processo inflamatório ou infeccioso 
que pudessem interferir nos procedimentos de coleta e alterações cognitivas. Os critérios de inclusão foram ter idade maior ou igual a 60 anos e idosos que não praticassem regularmente alguma modalidade de atividade física específica.

Os participantes da pesquisa assinaram o Termo de Consentimento Livre e Esclarecido anteriormente ao inicio da coleta. Todos os testes aplicados foram realizados no Laboratório de Estudos de Força (LABEF), nos períodos matutino e vespertino. Primeiramente, o avaliador, já no LABEF, realizava o mini exame do estado mental (MEEM), para excluir idosos que pudessem não compreender os testes. Pontuação menor que 13 para analfabetos, 18 para escolaridade baixa/média, e 26 para alta escolaridade foram destinadas como notas de corte. (BRUCKI et al., 2003; BERTOLUCCI et al., 1994). Posteriormente, os responsáveis pela coleta, preenchiam uma ficha padrão, contendo as informações sobre nome; estatura; peso; idade; histórico de quedas no último ano; histórico de quedas anterior ao último ano e um espaço para descrever como ocorreram cada um dos episódios de quedas. Foi realizada avaliação, através de resposta direta, para saber quantos episódios de quedas os sujeitos tiveram no ano anterior e antes de um ano. As medidas antropométricas foram coletadas através de estadiômetro e uma balança digital, devidamente calibrada. $\mathrm{Na}$ sequencia aplicou-se o Falls Efficacy Scale -
Internetional adaptado culturalmente para a população brasileira (FES-I-Brasil), um questionário com 16 itens que simulam atividades cotidianas da população idosa, onde devem relatar sua preocupação em cair, com cada resposta podendo receber de 1 a 4 pontos, assim como proposto por Camargos (2010), cuja finalidade é avaliar o medo de cair. Se a soma da pontuação for maior ou igual a 23, estas idosas são classificadas por risco de quedas esporádicas, maior que 31 , quedas recorrentes e abaixo de 23 sem risco. $\mathrm{O}$ teste de alcance funcional (TAF) seguiu os padrões de Duncan (1990), com as idosas devidamente descalças, em flexão de ombro a $90^{\circ}$, realizando um movimento de deslocamento anterior de tronco, simulando uma atividade extremamente funcional, e não sendo permitido rodar o tronco e nem retirar os pés do chão. Utilizou-se fita métrica neste teste. A escala de equilíbrio de Berg (EEB) foi utilizada da forma descrita por Berg (1989). O teste de alcance lateral (TAL), como proposto por Brauer (1999), segue o mesmo modelo do TAF, porém ao invés de fletirem o ombro, as idosas devem abduzi-lo a $90^{\circ}$ e realizar um movimento no sentido lateral. Não foi encontrado valor de estratificação para risco de quedas do TAL. Para a analise descritiva dos resultados de estratificação do risco de quedas foram utilizados dados de escores segundo diversos autores, como pode ser observado na tabela 1. Cada individuo realizou todos os testes em um mesmo dia e os dados 
foram anotados em planilhas de um computador, as coletas ocorreram entre fevereiro a abril de 2013. Três pesquisadores treinados foram responsáveis por todas as coletas. O trabalho foi aprovado pelo comitê de ética e pesquisa da universidade, sob o parecer 170.748, número de Certificado de Apresentação para Apreciação Ética (CAAE) 09216312.7.0000.0029.

Tabela 1 - Classificação da Estratificação do Risco de Quedas de Acordo Com Escores de Diferentes

Autores

\begin{tabular}{|c|c|c|c|}
\hline & $\begin{array}{l}\text { Pontuação de } \\
\text { Estratificação }\end{array}$ & $\mathrm{n}$ & $\%$ \\
\hline $\begin{array}{c}\text { FES-I-Brasil } \\
\text { por }\end{array}$ & $\begin{array}{c}\geq 23 \text { pontos } \\
\quad \text { (quedas }\end{array}$ & 11 & 26,8 \\
\hline \multirow{3}{*}{$\begin{array}{c}\text { Camargos } \\
\text { (2010) }\end{array}$} & esporádicas) & & \\
\hline & $\begin{array}{c}>31 \text { pontos } \\
\text { (quedas } \\
\text { recorrentes) }\end{array}$ & 4 & 9,8 \\
\hline & $\begin{array}{c}<23 \text { pontos } \\
\text { (sem risco } \mathrm{p} / \\
\text { quedas) }\end{array}$ & 26 & 63,4 \\
\hline TAF por & $\leq 15 \mathrm{~cm}$ & 40 & 97,6 \\
\hline $\begin{array}{c}\text { Duncan } \\
\text { (1990) }\end{array}$ & $\begin{array}{l}\text { (risco p/ } \\
\text { quedas) }\end{array}$ & 1 & 2,4 \\
\hline & $\begin{array}{c}>15 \mathrm{~cm} \text { (sem } \\
\text { risco } \mathrm{p} / \\
\text { quedas) }\end{array}$ & & \\
\hline TAF por & $\leq 17 \mathrm{~cm}$ & 40 & 97,6 \\
\hline Gai (2010) & $\begin{array}{l}\text { (risco p/ } \\
\text { quedas) }\end{array}$ & 1 & 2,4 \\
\hline & $\begin{array}{c}>17 \mathrm{~cm} \text { (sem } \\
\text { risco } \mathrm{p} / \\
\text { quedas) }\end{array}$ & & \\
\hline EBB por & $56-54$ pontos & 24 & 58,5 \\
\hline
\end{tabular}

Shumway-
Cook e

$\begin{array}{cccc}\text { Wollacott } & 53-46 \text { pontos } & 17 & 41,5 \\ \text { (2003) } & \text { (baixo a } & & \\ & \text { moderado } & & \\ & \text { risco p/ } & & \\ & \text { quedas) } & 0 & 0 \\ & <46 \text { pontos } & & \\ & \text { (alto risco p/ } & & \\ & \text { quedas) } & & \\ \text { EBB por } & >49 \text { pontos } & 39 & \\ \text { Shumway- } & \text { (sem risco p/ } & & \\ \text { Cook (1997) } & \text { quedas) } & 2 & 4,9 \\ & \leq 49 \text { pontos } & & \\ & \text { (risco p/ } & & \\ & \text { quedas) } & & \\ \text { EBB por } & >45 \text { pontos } & 41 & 100 \\ \text { Berg (1989) } & \text { (sem risco p/ } & & \\ & \text { quedas) } \\ & \leq 45 \text { (risco p/ } \\ & \text { quedas) } & & \end{array}$

FES-I-Brasil = Falls Efficacy Scale - Internetional adaptado culturalmente para a população brasileira; $\mathrm{TAF}=$ Teste de Alcance Funcional $; \mathrm{EBB}=$ Escala de Equilíbrio de Berg; $\mathrm{n}=$ Número de Indivíduos; \% = Porcentagem.

Fonte - Próprio autor

\section{Utilizou-se o software GraphPad InStat} para processamento e análise dos dados. As idosas foram divididas em dois grupos, as com quedas no último ano e as sem quedas no último ano. Então, realizou-se os testes Unpaired $t$ test e Mann-Whitney test para comparar todos os dados supracitados. Com relação aos dados de escores de estratificação de risco, realizou-se uma análise descritiva. Os testes de correlação de Spearman e de Pearson 
foram usados considerando-se toda a amostra.

Foram correlacionados os dados de idade, números de quedas (último ano e anterior), EEB e FES-I-Brasil. Adotou-se valores de significância para $\mathrm{p}<0,05$.

\section{RESULTADOS}

Ao final das coletas os grupos foram compostos por 18 idosas caidoras e 23 não caidoras. A caracterização da amostra total e separada em grupos pode ser observada na tabela 2. Observa-se ainda os valores obtidos nos históricos de quedas, testes funcionais e FES-I-Brasil, por todas as idosas e em grupos, além das comparações entre o grupo de caidoras e não caidoras.

Tabela 2 - Caracterização da Amostra e Resultado dos Testes de Comparação Entre Caidoras e Não Caidoras

\begin{tabular}{ccccc}
\hline & Caidoras & Não & P & Total \\
& & Caidoras & & \\
& Média \pm & & & Média \\
& DV & Média \pm & & \pm DV \\
& & DV & & \\
\hline Idade & $68,5 \pm$ & $66,78 \pm$ & 0,34 & $67,6 \pm$ \\
(anos) & 5,44 & 5,84 & & 5,6 \\
& & & & \\
Peso (Kg) & $60,32 \pm$ & $63,36 \pm$ & 0,38 & $62,1 \pm$ \\
& 11,38 & 7,72 & & 9,9 \\
Estatura & $1,53 \pm$ & $1,55 \pm$ & 0,32 & $1,5 \pm$ \\
(m) & 0,06 & 0,05 & & 0,1 \\
& & & & \\
IMC & $25,57 \pm$ & $26,34 \pm$ & 0,72 & $26,1 \pm$
\end{tabular}

$\begin{array}{ccccc}\left(\mathrm{Kg} / \mathrm{m}^{2}\right) & 4,26 & 3,61 & & 4 \\ & & & & \\ \text { Pontuação } & 52,7 \pm & 54,17 \pm & 0,08 & 53,6 \pm \\ \text { EEB } & 2,77 & 2,05 & & 2,5\end{array}$

$\begin{array}{ccccc}\text { TAF }(\mathrm{cm}) & 27,7 \pm & 32,2 \pm & <0,05^{*} & 30,2 \pm \\ & 5,5 & 5,6 & & 5,9 \\ \text { TAL }(\mathrm{cm}) & 20,36 \pm & 20,36 \pm & 0,99 & 20,4 \pm \\ & 4,96 & 5,45 & & 5,2 \\ \text { Pontuação } & 22,27 \pm & 23,91 \pm & 0,71 & 23,2 \pm \\ \text { FES-I- } & 5,68 & 8,13 & & 7,1 \\ \text { Brasil } & & & & \end{array}$

$\begin{array}{ccccc}\text { Número } & 1,44 \pm & - & - & 0,6 \pm \\ \text { de Quedas } & 0,70 & & & 0,9 \\ \text { no Último } & & & & \end{array}$

Ano

Número $\quad 0,44 \pm \quad 1 \pm 0,79 \quad 0,08 \quad 0,8 \pm 1$ de Quedas $\quad 1,19$ anterior ao

Último

Ano

FES-I-Brasil = Falls Efficacy Scale - Internetional adaptado culturalmente para a população brasileira; $\mathrm{TAF}=$ Teste de Alcance Funcional $; \mathrm{EBB}=$ Escala de Equilíbrio de Berg; * = p < 0,05; Kg = Quilograma; $\mathrm{m}$ $=$ metros $; \mathrm{Kg} / \mathrm{cm}^{2}=$ Quilograma por Centímetro Quadrado; DV = Desvio Padrão.

Fonte - Próprio autor

Na tabela 3 são apresentados os resultados dos testes de correlação entre idade, FES-IBrasil, testes funcionais e históricos de quedas passadas e recentes.

As comparações mostraram que existe uma diferença apenas nos resultados do TAF. O número de quedas anterior ao último ano e a 
EEB apresentaram, apenas, uma tendência a uma comparação diferente.

Ao correlacionar os valores de todos os parâmetros (exceto dados antropométricos) encontrou-se baixa correlação negativa ( $r=-$ 0,17) entre idade e EEB; Com relação ao número de quedas no último ano, verificou-se moderada correlação negativa $(r=-0,42)$ com o TAF. O TAF apresentou moderada correlação $(r=0,43)$ em relação a EEB. Do mesmo modo que a EEB teve correlação moderada $(\mathrm{r}=0,46)$ com TAL. As demais correlações não demostraram resultado significativo.

Tabela 3 - Resultados dos Testes de Correlação Entre as Variáveis do Grupo Total de Idosas

\begin{tabular}{|c|c|c|}
\hline & $\mathrm{r}$ & $\mathrm{p}$ \\
\hline $\begin{array}{l}\text { Idade e Número } \\
\text { de Quedas no } \\
\text { Último Ano }\end{array}$ & 0,03 & 0,26 \\
\hline $\begin{array}{c}\text { Idade e Número } \\
\text { de Quedas } \\
\text { Anterior ao } \\
\text { Último Ano }\end{array}$ & 0,04 & 0,2 \\
\hline $\begin{array}{c}\text { Idade e FES-I- } \\
\text { Brasil }\end{array}$ & $-0,17$ & 0,26 \\
\hline Idade e TAF & $-0,23$ & 0,16 \\
\hline Idade e TAL & $-0,25$ & 0,1 \\
\hline Idade e EEB & $-0,17$ & $<0,05^{*}$ \\
\hline Número de & 0,002 & 0,76 \\
\hline Quedas no & & \\
\hline Último Ano e & & \\
\hline Número de & & \\
\hline $\begin{array}{l}\text { Quedas Anterior } \\
\text { ao Último Ano }\end{array}$ & & \\
\hline Número de & 0,002 & 0,75 \\
\hline
\end{tabular}

Quedas no

Último Ano e

FES-I-Brasil

Número de

$-0,42$

$<0,05^{*}$

Quedas no

Último Ano e

TAF

Número de

$-0,0004$

0,99

Quedas no

Último Ano e

TAL

Número de

$-0,26$

0,09

Quedas no

Último Ano e

EEB

Número de

0,0001

0,93

Quedas Anterior

ao Último Ano e

FES-I-Brasil

Número de

0,03

0,83

Quedas Anterior

ao Último Ano e

TAF

Número de

0,09

0,2

Quedas Anterior

ao Último Ano e

TAL

Número de

0,04

0,2

Quedas Anterior

ao Último Ano e

EEB

FES-I-Brasil e

0,3

0,81

TAF

FES-I-Brasil e

$-0,21$

0,17

TAL

FES-I-Brasil e

0,0007

0,58

EEB

TAF e TAL $\quad 0,04 \quad 0,2$

TAF e EEB $\quad 0,43<0,05^{*}$

TAL e EEB $\quad 0,46<0,05^{*}$ 
FES-I-Brasil = Falls Efficacy Scale - Internetional adaptado culturalmente para a população brasileira; $\mathrm{TAF}=$ Teste de Alcance Funcional $; \mathrm{EBB}=$ Escala de Equilíbrio de Berg ; * $=\mathrm{p}<0,05$.

Fonte - Próprio autor

\section{DISCUSSÃO}

Dentre os testes de comparação entre caidoras e não caidoras, apenas, o TAF teve significância. Isto mostra que o teste citado teve uma diferença entre os grupos, caracterizando sua capacidade parar separar idosas nestes dois grupos. Por ser um teste que está relacionado com o equilíbrio postural, presume-se que possa haver uma diferença neste aspecto entre os dois grupos. Este resultado é contrário a Chiacchiero (2010), que não encontrou significância na comparação do TAF entre dois grupos de idosos com e sem episódios de quedas.

Foi verificada uma tendência a uma diferente comparação entre EEB, o que pode ser justificado da mesma forma que o TAF apresenta uma diferença significativa, pois ambos avaliam o equilíbrio postural.

A pesquisa de Wild (1981), concluiu que quedas antigas estão relacionadas à novos episódios, em idosos, ou seja, ao comparar o número de quedas antes de um ano entre caidoras e não caidoras, foi encontrada diferença. Porém, o presente estudo encontrou, apenas, uma tendência à este achado.

Não foi observado diferença na comparação entre o medo de cair. Achado semelhante ao de Walker (1990), onde concluiu que o medo de cair está de forma igual presente em grupos de idosas com e sem histórico de quedas. As demais variáveis não mostraram comparação significativa, o que demonstra a homogeneidade da amostra.

No atual estudo, apenas o escore da EEB descrito por Berg (1989) classificou 100\% das idosas como sem risco de quedas. As duas descrições do TAL classificaram, apenas, $2,4 \%$ das idosas como em risco de quedas.

Ao analisar as duas estratificação do TAF e todas da EEB, $19,51 \%$ apresentaram-se sem riscos. Já ao se observar a FES-I-Brasil e todas EEB sem risco, 39\% não estavam em risco.

Os achados das correlações da amostra demonstram que a idade e a pontuação da EEB possuem uma relação inversa, ou seja, enquanto uma apresenta valores baixos, a outra apresenta valores mais altos. Segundo Chiacchiero (2010), para se ter um bom equilíbrio é necessária a capacidade de reagir à estímulos externos, o que está relacionado dentre outras coisas com a força muscular. Porém, a sarcopenia está presente em idosos e aumenta com o passar dos anos, o que justifica a correlação encontrada. 
Houve correlação negativa do TAF com o número de quedas no último ano, dado capaz de demonstrar que quanto menor for a pontuação alcançada pelo individuo no TAF, mais suscetível à quedas esta pessoa estará. Indo contra este achado, Murphy (2003) observou que o teste em questão não foi um bom preditor de quedas em seu estudo, objetivado em identificar o melhor instrumento para mensurar prováveis eventos de quedas. É importante levar em consideração que o TAF do presente trabalho teve correlação com quedas no último ano e não foi preditor de quedas futuras, porém a amostra desta pesquisa era composta, exclusivamente, por idosas sedentárias, o que leva a acreditar que seus testes funcionais anteriores a um ano não apresentariam valores muito discrepantes dos atuais. Em contrapartida, o trabalho realizado por Duncan (1990) mostra como o TAF pode ser utilizado como teste capaz de avaliar a possível ocorrência de novas quedas.

Foi encontrada correlação estatisticamente significante entre TAF e EEB. Assim como TAF a EEB também é utilizada para avaliar o equilíbrio, porém, de forma mais completa, já que avalia diversas situações funcionais. A correlação destes dois parâmetros pode ser compreendida ao se observar os itens da EEB, ao analisá-los, percebe-se que o item número oito é exatamente igual ao TAF (DUNCAN et al., 1990; MIYAMOTO et al., 2004), o que explica o achado de uma pontuação maior na
EEB ser acompanhada de um TAF alto. Este dado vai ao encontro do estudo de Kakura (2011), que correlacionaram instrumentos de avaliação de risco de quedas em idosos comunitários, onde encontraram uma correlação moderada $(r=0,48)$ entre os testes mencionados.

O TAL é, provavelmente, dos instrumentos de avaliação do risco de quedas utilizados, o menos descrito na literatura. O que justifica a dificuldade de encontrar artigos relacionados ao assunto. Os parâmetros de TAL e EEB encontraram-se moderadamente correlacionados, demonstrando que ao aplicar estes testes em um idoso, valores altos devem ser encontrados em ambos, já que encontrouse uma relação de aumento proporcional entre eles. O TAL é capaz de avaliar o equilíbrio médio-lateral, que se encontra reduzido em mulheres idosas, e que está relacionado com quedas na terceira idade (NEWTON, 2001; NITZ; CHOY; ISLES, 2003). Esta correlação encontrada pode ser um valor que mostre a capacidade do TAL e da EEB de serem capazes de avaliar situações semelhantes de desempenho funcional. Newton (2001) encontrou resultado semelhante em seu estudo com 254 idosos da comunidade.

No presente estudo era esperado que houvesse correlação entre medo de cair e o histórico de quedas no ano anterior, porém esta hipótese não foi confirmada, mostrando que o teste não foi capaz de classificar as 
idosas dentro dos dois grupos. Com uma conclusão contraria a este achado, o estudo de Camargos (2010) relata que a pontuação final do FES-I-Brasil está mais relacionada, justamente, ao fator quedas no último ano.

Não foram encontradas correlações entre o medo de cair e nenhuma outra variável, como testes funcionais e históricos de quedas. Em contra partida, o trabalho de Vellas (1997) com 487 idosos comunitários concluiu que há uma relação entre quedas, medo de cair e a capacidade funcional. Estes achados de correlação que eram esperados ao início do estudo, podem não ter sido concretizados pela diferença do número de idosas entre os dois grupos.

Dentre os testes realizados, nenhum teve grandes dificuldades de ser realizado, porém muitas idosas apresentaram dificuldade em compreender e executar o TAF e o TAL, o que justifica a realização de um teste de adaptação e mais três para se conseguir uma avaliação adequada.

Os meios de se avaliar a autoeficácia de uma população idosa são bem descritos na literatura, com exceção do TAL. Poucos estudos foram encontrados sobre este teste na literatura, principalmente nacional. Seu escore de estratificação também não foi encontrado, pois seu estudo de validação não foi disponibilizado, em nenhuma base de dados.

Os testes funcionais realizados são capazes de avaliar o equilíbrio e riscos de quedas da população idosa (BERG et al., 1989; KAKURA; SILVA; NAVEGA, 2011), porém idosas com algumas limitações, tendem a entrar mais frequentemente nos grupos de risco de quedas. A amostra deste estudo foi constituída por idosas sem problemas físicos ou mentais, o que facilita a ocorrência do efeito teto em testes como a EEB. Na presente pesquisa $31,7 \%$ das idosas apresentaram pontuação máxima (56 pontos) no referido teste.

\section{CONCLUSÃO}

Ao dividir idosas em dois grupos, com e sem quedas recentes, o TAF foi o teste que melhor conseguiu diferenciar estes dois grupos, e sua correlação com quedas recentes, indica que dos testes usados é o melhor para identificar idosas com quedas recentes, apesar de ter classificado apensa 2,4\% dentro de um grupo com risco de quedas.

O estudo também leva a concluir que as avaliações do equilíbrio pelos TAF e TAL e pela EEB demonstram resultados semelhantes, porém um não deve substituir o outro, tendo em vista que analisam diferentes situações de equilíbrio postural dinâmico. $\mathrm{Na}$ presente pesquisa conclui-se que a idade pode interferir nos resultados da EEB, o que torna necessário utilizar valores de estratificação específicos 
para diferentes níveis de senescência, e quiçá de senilidade. Tendo sido observado que dentre todas as estratificações da EEB apenas duas idosas encontraram-se em risco de quedas, e sendo que foi observado o efeito teto em uma das estratificações.

\section{REFERÊNCIAS}

ARAÚJO, D. V.; OLIVEIRA, J. H. A.; BRACCO, O. L. Custo da Fratura

Osteoporótica de Fêmur no Sistema

Suplementar de Saúde Brasileiro. Arquivo Brasileiro de Endocrinologia e Metabologia. v.49, n.6, p. 897-901, 2005.

BERG, K.; WOOD-DAUPHINÉE, S.; WILLIAMS, J. I.; et al. Measuring balance in the elderly: preliminary development of an instrument. Physiotherapy Canada. v.41, n.6, p. 304-311, 1989.

BERTOLUCCI, P. H. F.; BRUCKI, S. M. D.; CAMPACCI, S. R.; et al. O mini-exame do estado mental em uma população geral: impacto da escolaridade. Arquivos de Neuropsiquiatria. v.52, n.1, p. 1-7, 1994.

BRAUER, S.; BURNS, Y.; GALLEY, P. Lateral Reach: a clinical measure of mediolateral postural stability. Physiotherapy Research International. v.4, n.2, p. 81-88, 1999.

BRUCKI, S. M. D.; NITRINI, R.; CARAMELLI, P.; et al. Sugestões para o uso do mini-exame do estado mental no Brasil. Arquivos de Neuropsiquiatria. v.61, n.3-B, p. 777-81, 2003.

CAMARGOS, F. F. O.; DIAS, J. M. D.;

FREIRE, M. T. F. Adaptação transcultural e avaliação das propriedades psicométricas da Falls Efficacy Scale - International em idosos brasileiros (FES-I-BRASIL). Revista

Brasileira de Fisioterapia. v.14, n.3, p. 237-43, 2010.

CHIACCHIERO, M.; DRESELY, B.; SILVA, U.; et al. The relationship between range of movement, flexibility, and balance in the elderly. Topics in Geriatric Rehabilitation. v.26, n.2, p. 147-154, 2010.

CHRISTOFOLETTI, G.; OLIANI, M. M.; GOBBI, L. T. B.; et al. Risco de Quedas em Idosos com Doença de Parkinson e Demência de Alzheimer: Um Estudo Transversal. Revista Brasileira de Fisioterapia. v.10, n.4, p. 429-33, 2006.

DOHERTY, T. J. Invited Review: Aging and sarcopenia. Journal of Applied Physiology. v.95, n.4, p. 1717-1727, 2003.

DUNCAN, P. W.; WEINER, D. K.; CHANDLER J.; et al. Functional reach. A new clinical measure of balance. Journal of Gerontology. v.45, n.6, p. 192-197, 1990.

FLETCHER, P. C.; HIRDES, J. P. Restriction in activity associated with fear of falling 
among community-based seniors using home care services. Age and Ageing. v.33, n.3, p. 273-279, 2004.

GAI, J.; GOMES, L.; NÓBREGA, O. T.; et al. Fatores associados a quedas em mulheres idosas residentes na comunidade. Revista da Associação Médica Brasileira. v.56, n. 3, p. 327-32, 2010.

GONÇALVES, L. H. T.; SILVA, A. H.; MAZO, G. Z.; et al. O idoso institucionalizado: avaliação da capacidade funcional e aptidão física. Caderno de Saúde Pública. v.26, n.9, p. 1738-1746, 2010.

GUIMARÃES, L. H. C. T.; GALDINO, D. C. A.; MARTINS, F. L. M.; et al. Comparação da propensão de quedas entre idosos que praticam atividade física e idosos sedentários. Revista Neurociência. v.12, n.2, p. 68-72, 2004.

HYATT, R. H.; WIGHTLAW, M.N.; BHAT, A.; et al. Association of muscle strength with functional status of elderly people. Age and Ageing. v.19, n.5, p. 330-336, 1990.

KAKURA, A. H.; SILVA, J. A. M. G.; NAVEGA, M. T. Análise da concordância entre instrumentos de avaliação do equilíbrio corporal em idosos. Revista Brasileira de Fisioterapia. v.15, n.6, p. 406-6, 2011.
LINDLE, R. S.; METTER, E. J.; LYNCH, N. A.; et al. Age and gender comparisons of muscle strength in 654 women and men aged 20-93 yr. Journal of Applied Physiology. v.83, n.5, p. 1581-1587, 1997.

MIYAMOTO, S. T.; JUNIOR, I. L.; BERG, K. O.; et al. Brazilian version of the Berg balance scale. Brazilian Journal of Medical and Biological Research. v.37, n.9, p. 1411-1421, 2004.

MURPHY, M. A.; OLSON, S. L.; PROTAS, E. J.; et al. Screening for falls in communitydwelling elderly. Journal of Aging and Physical Activity. v.11, n.1, p. 64-78, 2003.

NEWTON, R. A. Validity of the multidirectional reach test: a practical measure for limits of stability in older adults. Journal of Gerontology. v.56, n.4, p. M248-M252, 2001.

NITZ, J. C.; CHOY, N. L. L.; ISLES, R. C. Medial-lateral postural stability in communitydwelling women over 40 years of age. Department of Physical Therapy. v.17, n.7, p. 765-767, 2003.

NÓBREGA, A. C. L.; FREITAS, E. V.; OLIVEIRA, M. A. B; et al. Posicionamento oficial da sociedade brasileira de medicina do esporte e da sociedade brasileira de geriatria e gerontologia: atividade física e saúde do 
idoso. Revista Brasileira de Medicina do

Esporte. v.5, n.6, p. 207-211, 1999

OCHALA, J.; DORER, D. J.; FRONTERA,

W. R.; et al. Single skeletal muscle fiber

behavior after a quick stretch in young and

older men: a possible explanation of the

relative preservation of eccentric force in old age. European Journal of Physiology. v.452, n.4, p. 464-470, 2006.

PEREIRA, M. G. Epidemiologia: Teoria e prática. 12. ed. Rio de Janeiro: Guanabara Koogan, 2008.

ROSA, T. E. C.; BENÍCIO, M. H. A.; LATORRE, M. R. D. O.; et al. Fatores determinantes da capacidade funcional entre idosos. Revista de Saúde Pública da USP. v.37, n1, p. 40-8, 2003.

\section{SHUMWAY-COOK, A.; BALDWIN, M.;}

POLISSAR, N. L.; et al. Predicting the probability for fall in community-dwelling older adults. Department of Physical Therapy. v.77, n.8, p. 812-9, 1997.

SHUMWAY-COOK, A.; WOOLACOTT, M.
H. Controle motor: teoria e aplicações práticas. 2 ed. São Paulo: Manole, 2003. mecanismos e perspectivas. Ciência \& Saúde Coletiva. v.15, n.6, p. 2845-2857, 2010.

VELLAS, B. J.; WAYNE, S. J.; ROMERO, L. J.; et al. Fear of falling and restriction of mobility in elderly fallers. Age and Ageing. v. 26, n.3, p. 189-193, 1997.

WALKER, J. E.; HOWLAND, J. Falls and fear of falling among elderly persons linving in the community: occupational therapy interventions. The American Journal of Occupational Therapy. v.45, n.2, p. 119-122, 1990.

WALRAND, S.; GUILLET, C.; SALLES, J.; et al. Physiopathological mechanism of sarcopenia. Clinics in Geriatric Medicine. v.27, n.3, p. 365-385, 2011.

WILD, D.; NAYAK, U. S. L.; ISAACS, B. Prognosis of falls in old people at home. Journal of Epidemiology and Community Health. v.35, n.3, p. 200-204, 1981. 